\title{
Allis Clamp Fetal Scalp Stimulation
}

National Cancer Institute

\section{Source}

National Cancer Institute. Allis Clamp Fetal Scalp Stimulation. NCI Thesaurus. Code C114086.

A fetal stimulation technique that uses an Allis clamp applied transvaginally to the fetal head to elicit a fetal heart rate acceleration response during labor. 